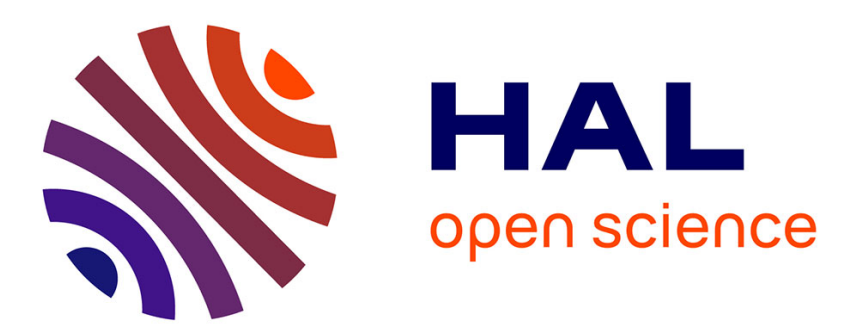

\title{
Robust Finite-time Stabilization and Observation of a Planar System Revisited
}

Andrey Polyakov, Yuri Orlov, Harshal Oza, Sarah Spurgeon

\section{To cite this version:}

Andrey Polyakov, Yuri Orlov, Harshal Oza, Sarah Spurgeon. Robust Finite-time Stabilization and Observation of a Planar System Revisited. 54th conference on Decision and Control 2015, IEEE, Dec 2015, Osaka, Japan. hal-01212582

\section{HAL Id: hal-01212582 \\ https://hal.inria.fr/hal-01212582}

Submitted on 6 Oct 2015

HAL is a multi-disciplinary open access archive for the deposit and dissemination of scientific research documents, whether they are published or not. The documents may come from teaching and research institutions in France or abroad, or from public or private research centers.
L'archive ouverte pluridisciplinaire HAL, est destinée au dépôt et à la diffusion de documents scientifiques de niveau recherche, publiés ou non, émanant des établissements d'enseignement et de recherche français ou étrangers, des laboratoires publics ou privés. 


\title{
Robust Finite-time Stabilization and Observation of a Planar System Revisited
}

\author{
Andrey Polyakov, Yuri Orlov, Harshal Oza and Sarah Spurgeon
}

\begin{abstract}
The second order planar nonlinear affine control problem is studied. A homogeneous robust finite-time stabilizing control is developed for the most general case of matched and, the more challenging, mismatched nonlinear perturbations. A homogeneous observer is designed for the planar system. Explicit restrictions on the observer gains and nonlinearities are presented. The main contribution lies in the proposed combination of the explicit and implicit Lyapunov function methods as well as weighted homogeneity while providing finitetime stability analysis. Theoretical results are supported by numerical simulations.
\end{abstract}

\section{INTRODUCTION}

The problem of finite-time stabilization goes back to the classical results of optimal control design [10]. Indeed, the time-optimal bang-bang algorithm represented in state feedback form is perhaps the most famous example of a finitetime stabilizing control. The first theoretical investigations of finite-time stability and stabilization appear in [26], where, in particular, the definition of finite-time stability as well as the corresponding Lyapunov theorems are developed for general dynamical systems. Subsequently, finite-time controllers are presented in [11]. Currently this topic is popular in the context of sliding mode [17], [18], [24], nonlinear [5], [6], [21], [23], robust [3], [12] and optimal [9], [25] control systems. The problem of finite-time stability also appears in mechanical systems with dry friction [1], [7].

Finite-time stability analysis is frequently related to the so-called homogeneity property of a dynamical system. Homogeneity theory has an established history going back to Euler and his homogeneous function theorem. Currently the so-called weighted homogeneity [5], [14], [18] has been introduced in the paper of V.I. Zubov [27]. It is wellknown [5], [14], [18] that asymptotically stable systems with negative homogeneity degree are finite-time stable. It is noteworthy that the problem of computing an upper bound on the finite settling time of homogeneous finite time systems without the help of finite time Lyapunov functions is an interesting problem. Recent results for the planar case can be found in [21] which makes use of a general result provided in [18].

Andrey Polyakov is with Inria Lille-Nord Europe, France (e-mail: andrey.polyakov@inria.fr) and Department of Control Systems and Informatics, ITMO University, Saint-Petersburg, Russia with the support of Grant 074-U01, Government of Russian Federation and Project 14.Z50.31.0031, Ministry of Education and Science of Russian Federation.

Yuri Orlov is with CICESE Research Center, Ensenada, Mexico.

Harshal Oza is with Institute of Engineering \& Technology, Ahmedabad University, India (formerly with University of Kent, UK).

Sarah Spurgeon is with the School of Engineering and Digital Arts, University of Kent at Canterbury, U.K.
Second order nonlinear models cover rather a large class of the physical (in particular, mechanical) and biological systems. This paper deals with finite-time control and estimation of planar systems. It revises results of [21] for the case of both matched and mismatched uncertainties. The principal contribution of this analysis is two fold. Firstly, a combination of the explicit and (homogeneity-based) implicit Lyapunov function analysis is presented for asymptotic and finite-time stability, respectively. It allows the control parameters and system nonlinearities to be quantitatively explored as well settling time estimates to be specified. Secondly, the upper bound on the unmatched disturbances for the double integrator system covers a broader class of disturbances with a non-Lipschitz upper bound. The paper is organized as follows. Section II presents the problem formulation followed by Sections III and IV which outline the controller and the observer respectively. Numerical examples are given in Section V. Section VI concludes the paper.

\section{Problem Statement}

Consider the system

$$
\left\{\begin{array}{l}
\dot{x}_{1}(t)=x_{2}(t)+\omega_{1}\left(t, x_{1}(t), x_{2}(t)\right), \\
\dot{x}_{2}(t)=u(t)+\omega_{2}\left(t, x_{1}(t), x_{2}(t)\right),
\end{array} \quad t \in \mathbb{R},\right.
$$

where $x_{1}, x_{2} \in \mathbb{R}$ are states, $u$ is the control input, $\omega_{1}$ : $\mathbb{R}^{3} \rightarrow \mathbb{R}$ and $\omega_{2}: \mathbb{R}^{3} \rightarrow \mathbb{R}$ are non-linear functions.

In this paper we deal with two problems:

- finite-time stabilization of the system (1) under the assumption that the nonlinear functions $\omega_{1}$ and $\omega_{2}$ are unknown but appropriately bounded;

- finite-time observation of the system (1) with the output

$$
y(t)=x_{1}(t)
$$

under the assumption that the nonlinear functions $\omega_{1}$ and $\omega_{2}$ are known.

Recent Lyapunov based results on finite-time control and observation for the perturbed double integrator can be found in [16], [17], [19]. The most recent robust finite time stabilization result when $\omega_{1}=0$ and $\left|\omega_{2}\right| \leq M\left|x_{2}\right|^{\alpha}$, for some a priori known constant $M>0$, can be found in the reference [20]. The results in [2], [21] present finite time stabilization for the case of matched non-Lipscitz perturbations while the result in [13] studies robustness to $\mathcal{C}^{1}$ matched disturbances, i.e. $\omega_{1}=0$ and $\omega_{2} \neq 0$. The paper [15] presents output feedback finite time stabilization for mismatched perturbations. It is noted in this reference that the output and not the whole state is finite time stabilized. In comparison, the proposed full state feedback based results in this paper are superior for the 
planar case as finite time stabilization of both the states $x_{1}(t)$ and $x_{2}(t)$ is achieved via the implicit Lyapunov function approach. Existing homogeneous ${ }^{1}$ controllers [4], [19] and homogeneous observers [22] are utilised in this paper. The main challenge is to achieve finite-time stabilization as well as observation of the system (1) in the presence of the perturbations $\omega_{1} \neq 0$ and $\omega_{2} \neq 0$.

\section{Finite-Time Controller}

Define the homogeneous feedback law [4], [19] as follows

$$
u(t)=-\mu_{1}\left|x_{1}(t)\right|^{\frac{\alpha}{2-\alpha}} \operatorname{sign}\left[x_{1}(t)\right]-\mu_{2}\left|x_{2}(t)\right|^{\alpha} \operatorname{sign}\left[x_{2}(t)\right],
$$

where $\alpha \in[0,1), \mu_{1}>0, \mu_{2}>0$ are control parameters. It is well-known [4, Th. 2], [18, Th. 3.1], that in the unperturbed case with $\omega_{1}=\omega_{2}=0$, the closed-loop system (1)-(3) is homogeneous of negative degree. Hence, asymptotic stability of the origin implies finite-time stability, i.e. the origin will be reached after some finite instant of time. The theorem below characterizes (possibly uncertain) functions $\omega_{1}$ and $\omega_{2}$, which do not destroy the finite-time stability property.

Theorem 1: If for some $\alpha \in[0,1)$ and $p_{i j} \geq 0, i, j=1,2$ the nonlinear functions $\omega_{1}$ and $\omega_{2}$ are bounded as follows

$$
\begin{aligned}
& \left|\omega_{1}\left(t, x_{1}, x_{2}\right)\right| \leq p_{11}\left|x_{1}\right|^{\frac{1}{2-\alpha}}+p_{12}\left|x_{2}\right| \\
& \left|\omega_{2}\left(t, x_{1}, x_{2}\right)\right| \leq p_{21}\left|x_{1}\right|^{\frac{\alpha}{2-\alpha}}+p_{22}\left|x_{2}\right|^{\alpha}
\end{aligned}
$$

for all $\left(t, x_{1}, x_{2}\right) \in \mathbb{R}^{3}$ and the control parameters $\mu_{1}>$ $0, \mu_{2}>0$ satisfies the restrictions

$$
\begin{gathered}
\frac{2 \mu_{1}-3 p_{11}-\left(\mu_{2}+p_{22}\right)(2-\alpha)}{2 \xi_{1}+\xi_{3}(\alpha+1)}>\frac{\left(\frac{2}{3-\alpha}\right)^{\frac{3-\alpha}{2}}}{\mu_{1}^{\frac{2-\alpha}{2}}}, \\
\frac{2 \mu_{1}-3 p_{11}-\left(\mu_{2}+p_{22}\right)(2-\alpha)}{2 \xi_{1}+\xi_{3}(\alpha+1)}>\frac{2+p_{11}+2 p_{21}+\alpha\left(\mu_{2}+p_{22}\right)}{2 \xi_{2}-\xi_{3}(1-\alpha)}>0 \\
\xi_{1}=\frac{(3-\alpha)\left(\mu_{1} p_{11}+\alpha\left(\mu_{1} p_{12}+p_{21}\right) /(\alpha+1)\right)}{2^{\frac{3-\alpha}{2}}\left(\mu_{1}(2-\alpha)\right)^{\frac{\alpha-1}{2}}} \\
\xi_{2}=\frac{(3-\alpha)\left(\mu_{2}-p_{22}-\left(\mu_{1} p_{12}+p_{21}\right) /(\alpha+1)\right)}{2^{\frac{3-\alpha}{2}}} \\
\xi_{3}=\left(\mu_{1}(2-\alpha)\right)^{\frac{\alpha-1}{2}} \xi_{1}
\end{gathered}
$$

then the feedback (3) stabilizes the system (1) to the origin in finite time with the settling time estimate (18).

Proofs of all the theorems are given in the Appendix, where settling-time estimates are also presented.

The nonlinear structure of the closed-loop system (1)-(3) implies a nonlinear restriction (5) to the control gains $\mu_{1}$ and $\mu_{2}$. Note that the system of nonlinear inequalities (5) is feasible for sufficiently small $p_{11} \geq 0$ and $p_{12} \geq 0$.

Consider some particular cases.

- If $p_{11}=p_{12}=p_{21}=0$ then the system (5) is feasible for any $p_{22} \geq 0$, since it is equivalent to $\mu_{1}>\left(\mu_{2}+\right.$ $\left.p_{22}\right)(2-\alpha) / 2$ and $\mu_{2}>p_{22}$. This coincides with the conditions derived in [20].

\footnotetext{
${ }^{1}$ A function $g: \mathbb{R}^{n} \rightarrow \mathbb{R}$ (resp. a vector field $f: \mathbb{R}^{n} \rightarrow \mathbb{R}^{n}$ ) is said to be $r$-homogeneous of degree $m$ iff for all $\lambda>0$ and for all $x \in \mathbb{R}^{n}$ we have $g(D(\lambda) x)=\lambda^{m} g(x)$ (resp. $f(D(\lambda) x)=\lambda^{m} D(\lambda) f(x)$ ), where $D(\lambda)=\operatorname{diag}\left\{\lambda^{r_{i}}\right\}_{i=1}^{n}$ and $r=\left(r_{1}, \ldots, r_{n}\right)^{T} \in \mathbb{R}_{+}^{n}$.
}

- In the case of matched perturbations $\left(p_{11}=p_{12}=0\right)$, it can be shown that

$$
\begin{gathered}
\mu_{2}>p_{22}+\frac{2-\alpha}{2} p_{21}, \\
\mu_{1}>\frac{2-\alpha}{2}\left(\mu_{2}+p_{22}\right)+\frac{\alpha p_{21}}{(3-\alpha)^{\frac{1-\alpha}{2}}}\left(\frac{(2-\alpha)^{\frac{1-\alpha}{2}}}{(\alpha+1) \sqrt{\mu_{1}}}+\frac{0.5}{\mu_{1}^{\frac{2-\alpha}{2}}}\right), \\
\frac{\mu_{1}-\frac{2-\alpha}{2}\left(\mu_{2}+p_{22}\right)}{\left(\mu_{1}(2-\alpha)\right)^{0.5(1-\alpha)}+0.5(\alpha+1)}>\frac{\alpha p_{21}\left(1+p_{21}+0.5 \alpha\left(\mu_{2}+p_{22}\right)\right)}{(\alpha+1)\left(\mu_{2}-p_{22}-0.5 p_{21}(2-\alpha)\right)} .
\end{gathered}
$$

The obtained system of inequalities is feasible with respect to $\mu_{1}, \mu_{2}$ for any $p_{21} \geq 0$ and $p_{22} \geq 0$.

- In the sliding mode case $(\alpha=0)$, the inequalities (4) become

$$
\begin{aligned}
& \left|\omega_{1}\left(t, x_{1}, x_{2}\right)\right| \leq p_{11}\left|x_{1}\right|^{\frac{1}{2}}+p_{12}\left|x_{2}\right|, \\
& \left|\omega_{2}\left(t, x_{1}, x_{2}\right)\right| \leq p_{21}+p_{22}
\end{aligned}
$$

and the inequalities (5) can be rewritten as follows

$$
\begin{gathered}
\mu_{1}>\mu_{2}+(3 / 2+0.5 / \sqrt{3}) p_{11}+p_{22}+p_{11} \frac{\sqrt{\mu_{1}}}{\sqrt{6}} \\
\mu_{2}>p_{21}+p_{22}+\mu_{1}\left(p_{12}+p_{11}\right) \\
\frac{\mu_{1}-\mu_{2}-\frac{3}{2} p_{11}-p_{22}}{\mu_{1} p_{11}\left(1+2^{\frac{3}{2}} \sqrt{\mu_{1}}\right)}>\frac{1+\frac{1}{2} p_{11}+p_{21}}{\mu_{2}-\mu_{1}\left(p_{12}+p_{11}\right)-p_{22}-p_{21}} .
\end{gathered}
$$

Evidently, the last system of inequalities is feasible for $p_{12}<1$ and sufficiently small $p_{11}<1-p_{12}$.

\section{FINITE-TIME OBSERVER}

Consider now the problem of finite-time observation of the system (1) with the measured output (2). Consider the so-called homogeneous observer [22] of the form

$$
\begin{aligned}
& \frac{d \hat{x}_{1}(t)}{d t}=-\nu_{1}\left|e_{1}(t)\right|^{\frac{1+\beta}{2}} \operatorname{sign}\left[e_{1}(t)\right]+\hat{x}_{2}(t)+\omega_{1}\left(t, y(t), \hat{x}_{2}(t)\right) \\
& \frac{d \hat{x}_{2}(t)}{d t}=-\nu_{2}\left|e_{1}(t)\right|^{\beta} \operatorname{sign}\left[e_{1}(t)\right]+\omega_{2}\left(t, y(t), \hat{x}_{2}(t)\right)
\end{aligned}
$$

where $\nu_{1}>0, \nu_{2}>0, \beta \in[0,1)$ and $e_{1}(t)=\hat{x}_{1}(t)-y(t)$. Denote $e_{2}(t)=\hat{x}_{2}(t)-x_{2}(t)$. The observation error equation has the form

$$
\begin{aligned}
& \dot{e}_{1}(t)=-\nu_{1}\left|e_{1}(t)\right|^{\frac{1+\beta}{2}} \operatorname{sign}\left[e_{1}(t)\right]+e_{2}(t)+\Delta \omega_{1}, \\
& \dot{e}_{2}(t)=-\nu_{2}\left|e_{1}(t)\right|^{\beta} \operatorname{sign}\left[e_{1}(t)\right]+\Delta \omega_{2},
\end{aligned}
$$

where $\Delta \omega_{1}=\omega_{1}\left(t, y(t), \hat{x}_{2}(t)\right)-\omega_{1}\left(t, y(t), x_{2}(t)\right)$ and $\Delta \omega_{2}=\omega_{2}\left(t, y(t), \hat{x}_{2}(t)\right)-\omega_{2}\left(t, y(t), x_{2}(t)\right)$.

Theorem 2: If for some $\beta \in[0,1), q_{1} \geq 0$ and $q_{2} \geq 0$ the inequalities

$$
\left|\Delta \omega_{1}\right| \leq q_{1}\left|e_{2}\right| \quad \text { and } \quad\left|\Delta \omega_{2}\right| \leq q_{2}\left|e_{2}\right|^{\frac{2 \beta}{1+\beta}}
$$

hold and the observer parameters $\nu_{1}>0$ and $\nu_{2}>0$ satisfy the restrictions

$$
\begin{gathered}
\frac{\frac{1}{2}-q_{1}-\frac{q_{2} \beta}{(1+\beta) \sqrt{\nu_{2}}}}{\sqrt{\nu_{2}}\left(\eta_{2}+\frac{1+3 \beta}{2(1+\beta)} \eta_{3}\right)}>\left(\frac{2(1+\beta)}{3+\beta}\right)^{\frac{3+\beta}{2(1+\beta)}}, \\
\frac{\frac{1}{2}-q_{1}-\frac{q_{2} \beta}{(1+\beta) \sqrt{\nu_{2}}}}{\sqrt{\nu_{2}}\left(\eta_{2}+\frac{1+3 \beta}{2(1+\beta)} \eta_{3}\right)}>\frac{\left(\sqrt{\nu_{2}}+\frac{\nu_{1}^{2}}{2 \sqrt{\nu_{2}}}+\frac{q_{2}}{1+\beta}\right)}{\left(\eta_{1}-\frac{1-\beta}{2(1+\beta)} \eta_{3}\right)}>0, \\
\eta_{1}=\frac{(3+\beta)}{2(1+\beta)^{\frac{3+\beta}{2(1+\beta)}}}\left(\nu_{1}-\frac{2 q_{1} \beta}{1+3 \beta}\right), \\
\eta_{2}=\frac{(3+\beta)}{2(1+\beta)\left(2 \nu_{2}\right)^{\frac{1-\beta}{2(1+\beta)}}}\left(\frac{q_{1}(1+\beta)}{1+3 \beta}+\frac{q_{2}}{\nu_{2}}\right), \\
\eta_{3}=\frac{(3+\beta)}{2(1+\beta)^{\frac{3+\beta}{2(1+\beta)}}}\left(\frac{q_{1}(1+\beta)}{1+3 \beta}+\frac{q_{2}}{\nu_{2}}\right) .
\end{gathered}
$$


then the system (7) is finite-time stable.

The system of inequalities (9) is feasible for sufficiently small $q_{1} \geq 0$ and $q_{2} \geq 0$. To estimate the upper bounds of the parameters $q_{1}$ and $q_{2}$, consider particular cases.

- If $q_{1}=q_{2}=0$, the inequalities (9) imply that the system (7) is finite time stable for any positive $\nu_{1}>0$ and $\nu_{2}>0$.

- If $q_{2}=0$ then the system of inequalities (9) is feasible for any $q_{1} \in[0,0.5)$.

- For $q_{1}=0$ the system (9) is feasible $\forall q_{2} \geq 0$.

\section{NUMERICAL EXAMPLES}

\section{A. Finite-time stabilization of the uncertain planar system}

Consider the nonlinear system of the form

$$
\left\{\begin{array}{l}
\dot{x}_{1}(t)=\frac{x_{1}}{4+4\left|x_{1}\right|}+\frac{x_{2}\left|x_{2}\right|}{8 \sqrt{x_{1}^{2}+x_{2}^{2}}}+x_{2}, \\
\dot{x}_{2}(t)=u(t),
\end{array}\right.
$$

where $x_{1}, x_{2} \in \mathbb{R}$. It is straightforward to see that the restriction (4) is satisfied for $\alpha=0$ with $p_{11}=p_{12}=1 / 8$ and $p_{21}=p_{22}=0$. Selecting $\mu_{1}=32$ and $\mu_{2}=24$, the conditions (5) hold. The simulation results are given in Fig. 1. The settling time obtained from numerical simulations is

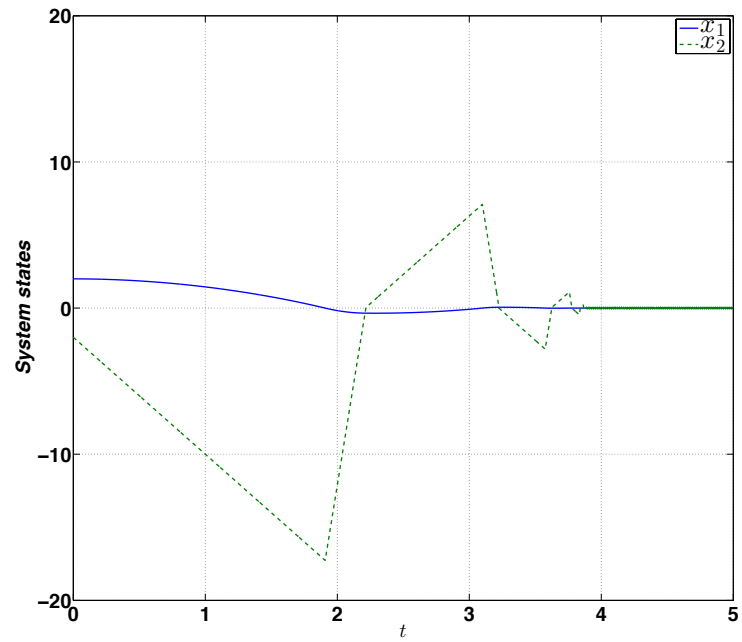

Fig. 1. Simulation results for finite-time stabilization

around 4. The analytical estimate of the settling time (see, the proof of Theorem 1, formula (18)) gives 8.4 that is conservative, although, less conservative than the latest result [21].

\section{B. Finite-time observation of the planar system}

Consider the non-linear positive system

$$
\left\{\begin{array}{l}
\dot{x}_{1}(t)=-2 x_{1}(t)+1-\frac{1}{2} x_{1}(t) x_{2}(t), \\
\dot{x}_{2}(t)=\left(\frac{1}{4}-x_{1}(t)\right) x_{2}^{\rho}(t),
\end{array}\right.
$$

where $x_{1} \geq 0$ and $x_{2} \geq 0$ are state variables, $\rho \in[0,1)$. The paper [8] utilizes this system with $\rho=1$ as the tumourimmune model for the so-called "steady control of tumour growth". In this case, the variable $x_{1}$ is related to the density of the lymphocyte population and $x_{2}$ is proportional to the density of the tumour cells. Due to physical restrictions, the variables are assumed to be bounded as follows $0<x_{1}^{\min } \leq$ $x_{1}(t) \leq x_{1}^{\max }$ and $0 \leq x_{2}(t) \leq x_{2}^{\max }$ for all $t \in \mathbb{R}$. It is assumed that the variable $x_{1}$ can only be measured and the problem is to reconstruct the state variable $x_{2}$ by means of the model (11).

Introduce a state observer of the form

$\frac{d \hat{x}_{1}(t)}{d t}=1-2 x_{1}(t)-\frac{x_{1}(t)}{2} \hat{x}_{2}(t)-\tilde{\nu}_{1}(t)\left|e_{1}(t)\right|^{\frac{1+\beta}{2}} \operatorname{sign}\left[e_{1}(t)\right]$, $\frac{d \hat{x}_{2}(t)}{d t}=\frac{1}{4} \hat{x}_{2}^{\rho}(t)-x_{1}(t) \hat{x}_{2}^{\rho}(t)+\tilde{\nu}_{2}(t)\left|e_{1}(t)\right|^{\beta} \operatorname{sign}\left[e_{1}(t)\right]$,

where $e_{1}(t)=\hat{x}_{1}(t)-x_{1}(t)$,

$$
\tilde{\nu}_{1}(t)=\frac{\nu_{1} x_{1}(t)}{2} \quad \text { and } \quad \tilde{\nu}_{2}(t)=\frac{\nu_{2} x_{1}(t)}{2},
$$

with $\nu_{1}>0$ and $\nu_{2}>0$. The change of time $\tau=\int_{0}^{t} \frac{2}{x_{1}(s)} d s$ allows the error equation to be expressed in the form

$$
\begin{aligned}
& \dot{e}_{1}(\tau)=e_{2}(\tau)-\nu_{1}\left|e_{1}(\tau)\right|^{\frac{1+\beta}{2}} \operatorname{sign}\left[e_{1}(\tau)\right], \\
& \dot{e}_{2}(\tau)=\frac{\left(4 x_{1}(\tau)-1\right)\left(\hat{x}_{2}^{\rho}(t)-x_{2}^{\rho}(t)\right)}{2 x_{1}(\tau)}-\nu_{2}\left|e_{1}(\tau)\right|^{\beta} \operatorname{sign}\left[e_{1}(\tau)\right],
\end{aligned}
$$

where $e_{2}=x_{2}(t)-\hat{x}_{2}(t)$ Therefore, the obtained error equation is equivalent to (7) with $\Delta \omega_{1}=0$ and $\left|\Delta \omega_{2}\right| \leq$ $q_{2} \cdot\left|e_{2}\right|^{\rho}$, where $q_{2}=\max _{x_{1} \in\left\{x_{1}^{\min }, x_{1}^{\max }\right\}}\left|2-\frac{1}{2 x_{1}}\right|$.

This satisfies the conditions of Theorem 2 if $\beta=\frac{\rho}{2-\rho}$. Note that for the case $\rho=1$, the finite-time homogeneous observer $\beta \in[0,1)$ can be constructed locally using the estimate of $\Delta \omega_{2}$ and a-priori boundedness of the state variables $x_{1}$ and $x_{2}$.

Fig. 2 presents simulation results for $\rho=0.5, x_{1}^{\min }=$ $0.1, x_{1}^{\max }=1$ and the parameters $\nu_{1}=10$ and $\nu_{2}=400$ satisfying (9).

\section{CONCLUSIONS}

Results on robust finite time stabilization are presented. A combination of homogeneous Lyapunov functions and implicit Lyapunov functions are utilised to prove finite time stability of planar uncertain system. Both the problems of finite time stable controller design and finite time stable observer design are analysed in the presence of mismatched disturbances that admit a non-Lipschitz upper bound. This is superior to existing results in the area. An interesting open problem is to identify similar Lyapunov functions to extend these results to more general $n$-dimensional system.

\section{APPENDIX}

Below Young's inequality is used whereby:

$$
\left|z_{1}\right|\left|z_{2}\right| \leq \frac{\left|z_{1}\right|^{r}}{r}+\frac{\left|z_{2}\right|^{q}}{q}, \quad \frac{1}{r}+\frac{1}{q}=1, \quad r, q>1,
$$

where $z_{1}, z_{2}$ are real numbers.

\section{A. Proof of Theorem 1}

\section{Asymptotic Stability (Explicit Lyapunov Method)}

Consider the Lyapunov function candidate

$$
V_{0}\left(x_{1}, x_{2}\right)=c U^{\frac{3-\alpha}{2}}\left(x_{1}, x_{2}\right)+\mu_{1}^{\frac{2-\alpha}{2}} x_{1} x_{2},
$$

where $c>0$ and

$$
U\left(x_{1}, x_{2}\right)=\mu_{1} \frac{2-\alpha}{2}\left|x_{1}\right|^{\frac{2}{2-\alpha}}+\frac{1}{2} x_{2}^{2} .
$$



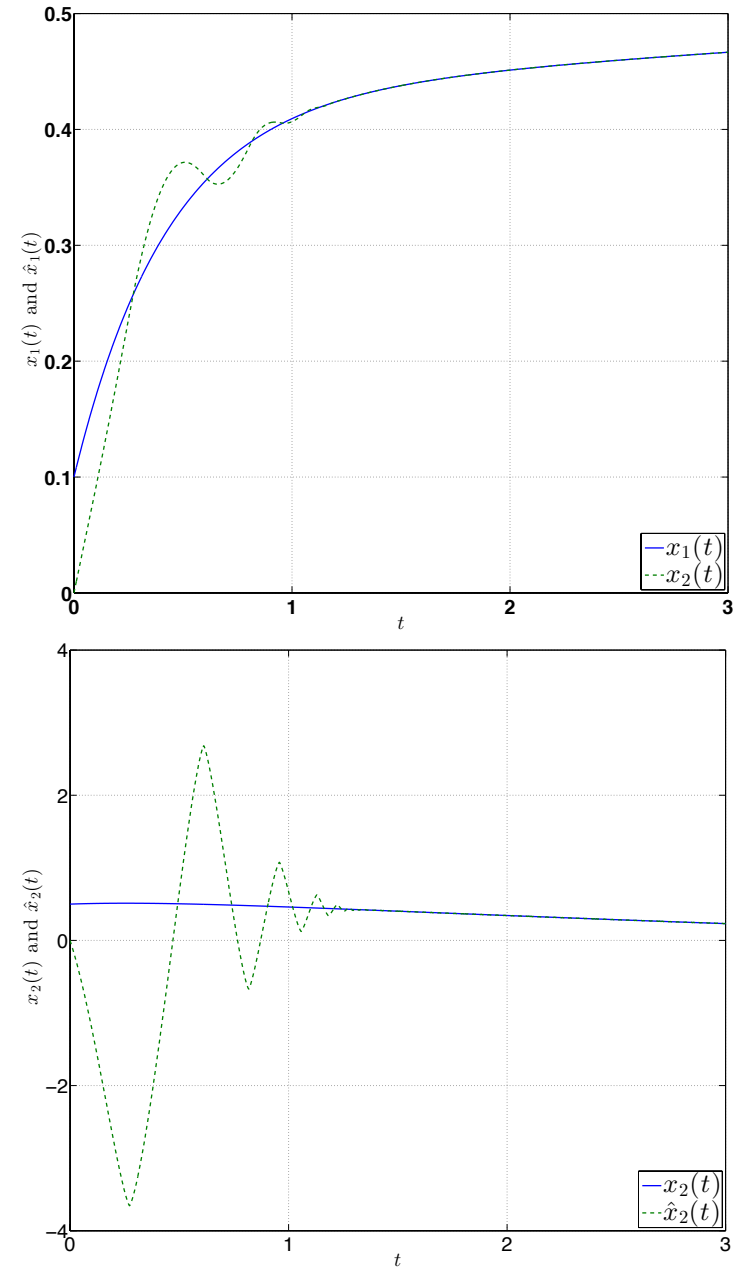

Fig. 2. Simulation results for finite-time observation

Applying Young's inequality for $q=3-\alpha$ and $r=\frac{3-\alpha}{2-\alpha}$ produces

$$
\left(\mu_{1}^{\frac{2-\alpha}{2}}\left|x_{1}\right|\right)^{\frac{2}{3-\alpha}}\left|x_{2}\right|^{\frac{2}{3-\alpha}} \leq \mu_{1} \frac{2-\alpha}{3-\alpha}\left|x_{1}\right|^{\frac{2}{2-\alpha}}+\frac{1}{3-\alpha} x_{2}^{2} .
$$

Hence, for

$$
c>\left(\frac{2}{3-\alpha}\right)^{\frac{3-\alpha}{2}}
$$

it follows that $V_{0}\left(x_{1}, x_{2}\right)>0$ outside the origin, i.e. $V_{0}$ is a positive definite function.

Since

$$
\begin{gathered}
\frac{d}{d t} U^{\frac{3-\alpha}{2}}\left(x_{1}, x_{2}\right)= \\
\frac{(3-\alpha)\left(\mu_{1}\left|x_{1}\right|^{\frac{\alpha}{2-\alpha}} \operatorname{sign}\left[x_{1}\right] \omega_{1}-\mu_{2}\left|x_{2}\right|^{\alpha+1}+x_{2} \omega_{2}\right) U^{\frac{1-\alpha}{2}}}{2} \leq \\
\frac{(3-\alpha)\left(\mu_{1} p_{11}\left|x_{1}\right|^{\frac{\alpha+1}{2-\alpha}}+\left(\mu_{1} p_{12}+p_{21}\right)\left|x_{1}\right|^{\frac{\alpha}{2-\alpha}}\left|x_{2}\right|-\left(\mu_{2}-p_{22}\right)\left|x_{2}\right|^{\alpha+1}\right) U^{\frac{1-\alpha}{2}}}{2}
\end{gathered}
$$

holds true, applying Young's inequality for $q=\alpha+1$ and $r=\frac{\alpha+1}{\alpha}$ produces

$$
\left|x_{1}\right|^{\frac{\alpha}{2-\alpha}}\left|x_{2}\right| \leq \alpha \frac{\left|x_{1}\right|^{\frac{\alpha+1}{2-\alpha}}}{\alpha+1}+\frac{\left|x_{2}\right|^{\alpha+1}}{\alpha+1} .
$$

The following may now be derived

$$
\begin{gathered}
\frac{d}{d t} U^{\frac{3-\alpha}{2}}\left(x_{1}, x_{2}\right) \leq-\frac{(3-\alpha)\left(\mu_{2}-p_{22}-\frac{\mu_{1} p_{12}+p_{21}}{\alpha+1}\right)}{2} U^{\frac{1-\alpha}{2}}\left|x_{2}\right|^{\alpha+1}+ \\
\frac{(3-\alpha)\left(\mu_{1} p_{11}+\alpha\left(\mu_{1} p_{12}+p_{21}\right) /(\alpha+1)\right)}{2} U^{\frac{1-\alpha}{2}}\left|x_{1}\right|^{\frac{\alpha+1}{2-\alpha}} \leq \\
-\xi_{2} x_{2}^{2}+\xi_{1}\left|x_{1}\right|^{\frac{2}{2-\alpha}}+\xi_{3}\left|x_{1}\right|^{\frac{\alpha+1}{2-\alpha}}\left|x_{2}\right|^{1-\alpha} \leq \\
-\left(\xi_{2}-0.5 \xi_{3}(1-\alpha)\right) x_{2}^{2}+\left(\xi_{1}+0.5 \xi_{3}(\alpha+1)\right)\left|x_{1}\right|^{\frac{2}{2-\alpha}}
\end{gathered}
$$

where

$$
\begin{gathered}
\xi_{1}=\frac{(3-\alpha)\left(\mu_{1} p_{11}+\alpha\left(\mu_{1} p_{12}+p_{21}\right) /(\alpha+1)\right)}{2^{\frac{3-\alpha}{2}}\left(\mu_{1}(2-\alpha)\right)^{\frac{\alpha-1}{2}}}, \\
\xi_{2}=\frac{(3-\alpha)\left(\mu_{2}-p_{22}-\left(\mu_{1} p_{12}+p_{21}\right) /(\alpha+1)\right)}{2^{\frac{3-\alpha}{2}}}, \\
\xi_{3}=\left(\mu_{1}(2-\alpha)\right)^{\frac{\alpha-1}{2}} \xi_{1},
\end{gathered}
$$

and Young's inequality is applied on the final step. On the other hand,

$$
\begin{gathered}
\frac{d}{d t}\left(x_{1} x_{2}\right)=x_{2}^{2}+x_{2} \omega_{1}-\mu_{1}\left|x_{1}\right|^{\frac{2}{2-\alpha}}-\mu_{2} x_{1}\left|x_{2}\right|^{\alpha} \operatorname{sign}\left[x_{2}\right]+x_{1} \omega_{2} \leq \\
\left(1+p_{21}\right) x_{2}^{2}+p_{11}\left|x_{1}\right|^{\frac{1}{2-\alpha}}\left|x_{2}\right|-\left(\mu_{1}-p_{11}\right)\left|x_{1}\right|^{\frac{2}{2-\alpha}}+ \\
\left(\mu_{2}+p_{22}\right)\left|x_{1}\right|\left|x_{2}\right|^{\alpha}
\end{gathered}
$$

Applying Young's inequality for $q=\frac{2}{\alpha}$ and $p=\frac{2}{2-\alpha}$

$$
\left|x_{1}\right|\left|x_{2}\right|^{\alpha} \leq \frac{2-\alpha}{2}\left|x_{1}\right|^{\frac{2}{2-\alpha}}+\frac{\alpha}{2}\left|x_{2}\right|^{2}
$$

and the inequality

$$
\left|x_{1}\right|^{\frac{1}{2-\alpha}}\left|x_{2}\right| \leq \frac{\left|x_{1}\right|^{\frac{2}{2-\alpha}}}{2}+\frac{x_{2}^{2}}{2}
$$

produce

$$
\begin{gathered}
\dot{V}_{0}\left(x_{1}, x_{2}\right) \leq \\
-\left(\mu_{1}^{\frac{2-\alpha}{2}}\left(\mu_{1}-\frac{3 p_{11}}{2}-\frac{\left(\mu_{2}+p_{22}\right)(2-\alpha)}{2}\right)-c \xi_{1}-\frac{c \xi_{3}(\alpha+1)}{2}\right)\left|x_{1}\right|^{\frac{2}{2-\alpha}} \\
-\left(c \xi_{2}-\frac{c \xi_{3}(1-\alpha)}{2}-\mu_{1}^{\frac{2-\alpha}{2}}\left(1+\frac{p_{11}}{2}+p_{21}+\frac{\alpha\left(\mu_{2}+p_{22}\right)}{2}\right)\right) x_{2}^{2} .
\end{gathered}
$$

Therefore, if the inequalities (15) and

$$
\frac{\mu_{1}-\frac{3 p_{11}}{2}-\frac{\left(\mu_{2}+p_{22}\right)(2-\alpha)}{2}}{\xi_{1}+\frac{\xi_{3}(\alpha+1)}{2}}>\frac{c}{\mu_{1}^{\frac{2-\alpha}{2}}}>\frac{1+\frac{p_{11}}{2}+p_{21}+\frac{\alpha\left(\mu_{2}+p_{22}\right)}{2}}{\xi_{2}-\frac{\xi_{3}(1-\alpha)}{2}}
$$

hold then the function (13) is a strict Lyapunov function for the closed-loop system (1). In the sliding mode case $(\alpha=0)$ then the right-hand side of the closed-loop system is discontinuous. The Lyapunov function-based stability analysis can be provided in this case using the concept of Filippov solutions (see, for example, [18]).

II. Finite-time Stability (Implicit Lyapunov Method)

The function $V_{0}\left(x_{1}, x_{2}\right)$ is $r$-homogeneous of degree $\frac{3-\alpha}{1-\alpha}$ with the weights: $r_{1}=\frac{2-\alpha}{1-\alpha}$ and $r_{2}=\frac{1}{1-\alpha}$. Indeed, $V_{0}\left(\lambda^{r_{1}} x_{1}, \lambda^{r_{2}} x_{2}\right)=\lambda^{\frac{3-\alpha}{1-\alpha}} V_{0}\left(x_{1}, x_{2}\right), \forall x_{1}, x_{2} \in \mathbb{R}$ and $\forall \lambda>0$.

Let us denote $\Omega=\left\{\left(z_{1}, z_{2}\right) \in \mathbb{R}^{2}: V_{0}\left(z_{1}, z_{2}\right)=1\right\}$ and $\gamma=\inf _{\left(z_{1}, z_{2}\right) \in \Omega} l_{1}\left|z_{1}\right|^{\frac{2}{2-\alpha}}+l_{2} z_{2}^{2}$, where

$$
\begin{gathered}
l_{1}=\mu_{1}^{\frac{2-\alpha}{2}}\left(\mu_{1}-\frac{3 p_{11}}{2}-\frac{\left(\mu_{2}+p_{22}\right)(2-\alpha)}{2}\right)-c \xi_{1}-\frac{c \xi_{3}(\alpha+1)}{2}>0 \\
l_{2}=c \xi_{2}-\frac{c \xi_{3}(1-\alpha)}{2}-\mu_{1}^{\frac{2-\alpha}{2}}\left(1+\frac{p_{11}}{2}+p_{21}+\frac{\alpha\left(\mu_{2}+p_{22}\right)}{2}\right)>0 .
\end{gathered}
$$


In this case, $\dot{V}_{0}\left(x_{1}, x_{2}\right) \leq-\gamma<0$ if $\left(x_{1}, x_{2}\right) \in \Omega$.

The finite-time Lyapunov function will be designed using the implicit Lyapunov function method:

Theorem [23]

If there exists a continuous function

$$
\begin{gathered}
Q: \mathbb{R}_{+} \times \mathbb{R}^{n} \rightarrow \mathbb{R} \\
(V, x) \rightarrow Q(V, x)
\end{gathered}
$$

that is

C1) continuously differentiable in the domain $\mathbb{R}_{+} \times \mathbb{R}^{n}$;

$C 2$ ) for any $x \in \mathbb{R}^{n} \backslash\{0\}$ there exist $V^{-} \in \mathbb{R}_{+}$and $V^{+} \in \mathbb{R}_{+}$:

$$
Q\left(V^{-}, x\right)<0<Q\left(V^{+}, x\right) ;
$$

C3) for $\Omega=\left\{(V, x) \in \mathbb{R}^{n+1}: Q(V, x)=0\right\}$ the limits

$$
\lim _{\substack{x \rightarrow 0 \\(V, x) \in \Omega}} V=0, \lim _{\substack{V \rightarrow 0^{+} \\(V, x) \in \Omega}}\|x\|=0, \lim _{\substack{x \rightarrow \infty \\(V, x) \in \Omega}} V=+\infty ;
$$

exist;

C4) for $\forall V \in \mathbb{R}_{+}$and $\forall x \in \mathbb{R}^{n} \backslash\{0\}$ the inequality

$$
-\infty<\frac{\partial Q(V, x)}{\partial V}<0
$$

holds;

C5) $\forall t \in \mathbb{R}_{+}, \forall V \in \mathbb{R}_{+}, \forall x \in \mathbb{R}^{n} \backslash\{0\}: Q(V, x)=0$

$$
\frac{\partial Q(V, x)}{\partial x} f(x) \leq c V^{1-\mu} \frac{\partial Q(V, x)}{\partial V}
$$

where $c>0$ and $0<\mu \leq 1$ are some constants, then the equilibrium point $x=0$ of the system

$$
\dot{x}=f(x)
$$

is globally finite time stable with the following settling time estimate

$$
T\left(x_{0}\right) \leq \frac{V^{\mu}\left(x_{0}\right)}{c \mu},
$$

where $V(x): Q(V, x)=0$.

In order to apply the implicit Lyapunov function theorem to the system (1), define

$$
Q(V, x)=V_{0}\left(V^{-r_{1}} x_{1}, V^{-r_{2}} x_{2}\right)-1,
$$

where $x=\left(x_{1}, x_{2}\right)^{T} \in \mathbb{R}^{2}, r_{1}=\frac{2-\alpha}{1-\alpha}, r_{2}=\frac{1}{1-\alpha}$ are homogeneity weights of the system (1). It can be easily checked that the conditions $\mathrm{C} 1$ )-C4) hold. On the one hand,

$$
\frac{\partial Q}{\partial V}=V_{0}\left(x_{1}, x_{2}\right) \frac{\partial}{\partial V} V^{-\frac{3-\alpha}{1-\alpha}}=-\frac{3-\alpha}{1-\alpha} \frac{V_{0}\left(\frac{x_{1}}{V^{r_{1}}}, \frac{x_{2}}{V^{r_{2}}}\right)}{V}=-\frac{3-\alpha}{1-\alpha} \frac{1}{V}
$$

if $Q(V, x)=0$.

On the other hand, for the system (1)

$$
\begin{aligned}
& \frac{\partial Q}{\partial x} f(x)=V^{-\frac{3-\alpha}{1-\alpha}} \dot{V}_{0}\left(x_{1}, x_{2}\right) \leq-V^{-\frac{3-\alpha}{1-\alpha}}\left(l_{1}\left|x_{1}\right|^{\frac{2}{2-\alpha}}+l_{2} x_{2}^{2}\right)= \\
& -V^{-1}\left(l_{1}\left|V^{-r_{1}} x_{1}\right|^{\frac{2}{2-\alpha}}+l_{2}\left(V^{-r_{2}} x_{2}\right)^{2}\right) \leq-\gamma V^{-1} \text { if } Q(V, x)=0 .
\end{aligned}
$$

Therefore, the condition C5) also holds for $\mu=1$ and $c=$ $\frac{\gamma(1-\alpha)}{3-\alpha}$ and the settling time admits the following estimate:

$$
T(x) \leq \frac{3-\alpha}{\gamma(1-\alpha)} V_{0}^{\frac{1-\alpha}{3-\alpha}}\left(x_{1}, x_{2}\right) .
$$

III. Estimation of $\gamma$. If $z_{2} \neq 0$ then $V_{0}\left(z_{1}, z_{2}\right)=1$ can be rewritten as follows

$$
c\left(\frac{\mu_{1}(2-\alpha)}{2} \frac{\left|z_{1}\right|^{\frac{2}{2-\alpha}}}{z_{2}^{2}}+\frac{1}{2}\right)^{\frac{3-\alpha}{2}}+\left(\mu_{1} \frac{\left|z_{1}\right|^{\frac{2}{2-\alpha}}}{z_{2}^{2}}\right)^{\frac{2-\alpha}{2}} \operatorname{sign}\left[z_{1} z_{2}\right]=\left|z_{2}\right|^{\alpha-3}
$$

Hence, denoting $y_{1}=\frac{\left|z_{1}\right|^{\frac{2}{2-\alpha}}}{z_{2}^{2}}$ and $y_{2}=\left|z_{2}\right|^{3-\alpha}$ allows the following derivation:

$$
\gamma=\inf _{y_{1} \geq 0} \frac{l_{1} y_{1}+l_{2}}{\left(c\left(\frac{\mu_{1}(2-\alpha)}{2} y_{1}+\frac{1}{2}\right)^{\frac{3-\alpha}{2}}+\mu_{1}^{\frac{2-\alpha}{2}} y_{1}^{\frac{2-\alpha}{2}}\right)^{\frac{2}{3-\alpha}}}
$$

In the general case, the parameter $\gamma$ can be calculated numerically. However, if $\frac{l_{2}}{l_{1}} \leq \frac{1}{\mu_{1}(2-\alpha)}$ then the function under inf is monotone decreasing by $y_{1} \in[0,+\infty)$ and

$$
\gamma=\frac{2 l_{1}}{\mu_{1}(2-\alpha) c^{\frac{2}{3-\alpha}}} \text {. }
$$

\section{B. Proof of Theorem 2}

\section{Asymptotic Stability (Explicit Lyapunov Method)}

Consider the Lyapunov function candidate

$$
V_{0}\left(e_{1}, e_{2}\right)=c U^{\frac{3+\beta}{2(1+\beta)}}\left(e_{1}, e_{2}\right)-\frac{1}{\sqrt{\nu_{2}}} e_{1} e_{2},
$$

where $c>0$ and

$$
U\left(e_{1}, e_{2}\right)=\frac{1}{\beta+1}\left|e_{1}\right|^{\beta+1}+\frac{1}{2 \nu_{2}} e_{2}^{2} .
$$

Applying Young's inequality for $r=\frac{3+\beta}{2}$ and $q=\frac{3+\beta}{1+\beta}$ it can be derived that

$$
\left|x_{1}\right|^{\frac{2(1+\beta)}{3+\beta}}\left(\frac{\left|x_{2}\right|}{\sqrt{\nu_{2}}}\right)^{\frac{2(1+\beta)}{3+\beta}} \leq \frac{2}{3+\beta}\left|x_{1}\right|^{1+\beta}+\frac{1+\beta}{(3+\beta) \nu_{2}} x_{2}^{2} .
$$

Therefore, for

$$
c>\left(\frac{2(1+\beta)}{3+\beta}\right)^{\frac{3+\beta}{2(1+\beta)}}
$$

the function $V_{0}$ is positive definite.

Since

$$
\begin{gathered}
\frac{d}{d t} U^{\frac{3+\beta}{2(1+\beta)}}\left(e_{1}, e_{2}\right)=\frac{3+\beta}{2(1+\beta)} U^{\frac{1-\beta}{2(1+\beta)}} \dot{U}= \\
\frac{(3+\beta) U^{\frac{1-\beta}{2(1+\beta)}}}{2(1+\beta)}\left(-\nu_{1}\left|e_{1}\right|^{\frac{1+3 \beta}{2}}+\Delta \omega_{1}\left|e_{1}\right|^{\beta} \operatorname{sign}\left[e_{1}\right]+\frac{e_{2} \Delta \omega_{2}}{\nu_{2}}\right) \leq \\
\frac{(3+\beta) U^{\frac{1-\beta}{2(1+\beta)}}}{2(1+\beta)}\left(-\nu_{1}\left|e_{1}\right|^{\frac{1+3 \beta}{2}}+q_{1}\left|e_{1}\right|^{\beta}\left|e_{2}\right|+\frac{q_{2}\left|e_{2}\right|^{\frac{1+3 \beta}{1+\beta}}}{\nu_{2}}\right),
\end{gathered}
$$

then applying Young's inequality for $r=\frac{1+3 \beta}{2 \beta}, q=\frac{1+3 \beta}{1+\beta}$

$$
\left|e_{1}\right|^{\beta}\left|e_{2}\right| \leq \frac{2 \beta}{1+3 \beta}\left|e_{1}\right|^{\frac{1+3 \beta}{2}}+\frac{1+\beta}{1+3 \beta}\left|e_{2}\right|^{\frac{1+3 \beta}{1+\beta}}
$$

it follows that

$$
\begin{gathered}
\frac{d}{d t} U^{\frac{3+\beta}{2(1+\beta)}}\left(e_{1}, e_{2}\right) \leq \frac{(3+\beta)}{2(1+\beta)} U^{\frac{1-\beta}{2(1+\beta)}} \times \\
\left(-\left(\nu_{1}-\frac{2 q_{1} \beta}{1+3 \beta}\right)\left|e_{1}\right|^{\frac{1+3 \beta}{2}}+\left(\frac{q_{1}(1+\beta)}{1+3 \beta}+\frac{q_{2}}{\nu_{2}}\right)\left|e_{2}\right|^{\frac{1+3 \beta}{1+\beta}}\right) \leq \\
-\eta_{1}\left|e_{1}\right|^{1+\beta}+\eta_{2}\left|e_{2}\right|^{2}+\eta_{3}\left|e_{1}\right|^{\frac{1-\beta}{2}}\left|e_{2}\right|^{\frac{1+3 \beta}{1+\beta}},
\end{gathered}
$$

where

$$
\eta_{1}=\frac{(3+\beta)\left(\nu_{1}-\frac{2 q_{1} \beta}{1+3 \beta}\right)}{2(1+\beta)^{\frac{3+\beta}{2(1+\beta)}}}, \eta_{3}=\frac{(3+\beta)\left(\frac{q_{1}(1+\beta)}{1+3 \beta}+\frac{q_{2}}{\nu_{2}}\right)}{2(1+\beta)^{\frac{3+\beta}{2(1+\beta)}}},
$$




$$
\eta_{2}=\frac{(3+\beta)}{2(1+\beta)\left(2 \nu_{2}\right)^{\frac{1-\beta}{2(1+\beta)}}}\left(\frac{q_{1}(1+\beta)}{1+3 \beta}+\frac{q_{2}}{\nu_{2}}\right),
$$

Applying again Young's inequality for $r=\frac{2(1+\beta)}{1-\beta}, q=\frac{2(1+\beta)}{1+3 \beta}$ it is obtained that $\left|e_{1}\right|^{\frac{1-\beta}{2}}\left|e_{2}\right|^{\frac{1+3 \beta}{1+\beta}} \leq \frac{1-\beta}{2(1+\beta)}\left|e_{1}\right|^{1+\beta}+\frac{1+3 \beta}{2(1+\beta)} e_{2}^{2}$, $\frac{d U^{\frac{3+\beta}{2(1+\beta)}}\left(e_{1}, e_{2}\right)}{d t} \leq-\left(\eta_{1}-\frac{(1-\beta) \eta_{3}}{2(1+\beta)}\right)\left|e_{1}\right|^{1+\beta}+\left(\eta_{2}+\frac{(1+3 \beta) \eta_{3}}{2(1+\beta)}\right) e_{2}^{2}$

On the other hand,

$\frac{d}{d t}\left(-\frac{e_{1} e_{2}}{\sqrt{\nu_{2}}}\right) \leq-\frac{1-q_{1}}{\sqrt{\nu_{2}}} e_{2}^{2}+\frac{\nu_{1}\left|e_{1}\right|^{\frac{1+\beta}{2}}\left|e_{2}\right|+q_{2}\left|e_{1}\right|\left|e_{2}\right|^{\frac{2 \beta}{1+\beta}}}{\sqrt{\nu_{2}}}+\sqrt{\nu_{2}}\left|e_{1}\right|^{1+\beta}$ and applying Young's inequality for $r=1+\beta, q=\frac{1+\beta}{\beta}$ it is obtained that $\left|e_{1}\right|\left(\nu_{2}^{-1 / 2}\left|e_{2}\right|^{\frac{2 \beta}{1+\beta}}\right) \leq \frac{\left|e_{1}\right|^{1+\beta}}{1+\beta}+\frac{\beta}{1+\beta} e_{2}^{2} \nu_{2}^{-1}$,

$\frac{d}{d t}\left(-\frac{e_{1} e_{2}}{\sqrt{\nu_{2}}}\right) \leq-\frac{\frac{1}{2}-q_{1}-\frac{q_{2} \beta}{(1+\beta) \sqrt{\nu_{2}}}}{\sqrt{\nu_{2}}} e_{2}^{2}+\left(\sqrt{\nu_{2}}+\frac{\nu_{1}^{2}}{2 \sqrt{\nu_{2}}}+\frac{q_{2}}{1+\beta}\right)\left|e_{1}\right|^{1+\beta}$

Hence,

$$
\begin{gathered}
\dot{V}_{0}\left(e_{1}, e_{2}\right) \leq-\left(\frac{\frac{1}{2}-q_{1}-\frac{q_{2} \beta}{(1+\beta) \sqrt{\nu_{2}}}}{\sqrt{\nu_{2}}}-c\left(\eta_{2}+\frac{1+3 \beta}{2(1+\beta)} \eta_{3}\right)\right) e_{2}^{2} \\
-\left(c\left(\eta_{1}-\frac{1-\beta}{2(1+\beta)} \eta_{3}\right)-\left(\sqrt{\nu_{2}}+\frac{\nu_{1}^{2}}{2 \sqrt{\nu_{2}}}+\frac{q_{2}}{1+\beta}\right)\right)\left|e_{1}\right|^{1+\beta} .
\end{gathered}
$$

Hence, the inequality (21) and the inequalities

$$
\frac{\frac{1}{2}-q_{1}-\frac{q_{2} \beta}{(1+\beta) \sqrt{\nu_{2}}}}{\sqrt{\nu_{2}}\left(\eta_{2}+\frac{1+3 \beta}{2(1+\beta)} \eta_{3}\right)}>c>\frac{\left(\sqrt{\nu_{2}}+\frac{\nu_{1}^{2}}{2 \sqrt{\nu_{2}}}+\frac{q_{2}}{1+\beta}\right)}{\left(\eta_{1}-\frac{1-\beta}{2(1+\beta)} \eta_{3}\right)}
$$

guarantee that $V_{0}$ is a strict Lyapunov function for (7).

II. Finite-time Stability (Implicit Lyapunov Method) The function $V_{0}\left(x_{1}, x_{2}\right)$ is $r$-homogeneous with the same weights as the original system : $r_{1}=\frac{2}{1-\beta}$ and $r_{2}=\frac{1+\beta}{1-\beta}$. Namely, $V_{0}\left(\lambda^{r_{1}} x_{1}, \lambda^{r_{2}} x_{2}\right)=\lambda^{\frac{3+\beta}{1-\beta}} V_{0}\left(x_{1}, x_{2}\right)$ for all $x_{1} \in$ $\mathbb{R}, x_{2} \in \mathbb{R}$ and $\lambda>0$.

Denote $\Omega=\left\{\left(z_{1}, z_{2}\right) \in \mathbb{R}^{2}: V_{0}\left(z_{1}, z_{2}\right)=1\right\}$ and $\gamma=\inf _{\left(z_{1}, z_{2}\right) \in \Omega} l_{1}\left|z_{1}\right|^{\frac{2}{2-\alpha}}+l_{2} z_{2}^{2}>0$, where $l_{1}=$ $c\left(\eta_{1}-\frac{1-\beta}{2(1+\beta)} \eta_{3}\right)-\left(\sqrt{\nu_{2}}+\frac{\nu_{1}^{2}}{2 \sqrt{\nu_{2}}}+\frac{q_{2}}{1+\beta}\right)>0$ and $l_{2}=$ $\frac{\frac{1}{2}-q_{1}-\frac{q_{2} \beta}{(1+\beta) \sqrt{\nu_{2}}}}{\sqrt{\nu_{2}}}-c\left(\eta_{2}+\frac{1+3 \beta}{2(1+\beta)} \eta_{3}\right)>0$. In this case, $\dot{V}_{0}\left(x_{1}, x_{2}\right) \leq-\gamma<0$ if $\left(x_{1}, x_{2}\right) \in \Omega$.

The proof of finite-time stability can be provided by means of the implicit Lyapunov function method similarly to Theorem 1 and the settling time estimate has the form

$$
T(x) \leq \frac{3+\beta}{\gamma(1-\beta)} V_{0}^{\frac{1-\beta}{3+\beta}}\left(x_{1}, x_{2}\right) .
$$

\section{REFERENCES}

[1] V. Acary and B. Brogliato. Numerical Methods for Nonsmooth Dynamical Systems, volume 35 of Lecture Notes in Applied and Computational Mechanics. Springer Verlag, 2008.

[2] V. Andrieu, L. Praly, and A. Astolfi. Homogeneous approximation, recursive observer design, and output feedback. SIAM Journal on Control \& Optimization, 47(4):1814 - 1850, 2008.

[3] E. Bernuau, D. Efimov, W Perruquetti, and A. Polyakov. On homogeneity and its application in sliding mode control. Journal of The Franklin Institute, 351(4):1866-1901, 2014.

[4] S. Bhat and D.S. Bernstein. Finite-time stability of homogeneous systems. In Proceedings of the American Control Conference, volume 4, pages 2513-2514, Jun 1997.
[5] S. P. Bhat and D. S. Bernstein. Geometric homogeneity with applications to finite-time stability. Mathematics of Control, Signals and Systems, 17:101-127, 2005.

[6] S.P. Bhat and D.S. Bernstein. Finite-time stability of continuous autonomous systems. SIAM Journal of Control and Optimization, 38(3):751-766, 2000.

[7] J.J.B. Biemond, N. van de Wouw, and H. Nijmeijer. Bifurcations of equilibrium sets in mechanical systems with dry friction. Physica $D$ : Nonlinear Phenomena, 241(22):1882-1894, 2012.

[8] C. Cattani and A. Ciancio. Qualitative analysis of second-order models ot tumor-immune system competition. Mathematical and Computer Modelling, 47:1339-1355, 2008.

[9] F.L. Chernousko, I.M. Ananevskii, and S.A. Reshmin. Control of nonlinear dynamical systems: methods and applications. Berlin: Springer-Verlag, 2008.

[10] A. Fuller. Relay control systems optimized for various performance criteria. In Proceedings of the 1st IFAC Triennial World Congress, pages $510-519,1960$.

[11] V.T. Haimo. Finite time controllers. SIAM Journal of Control and Optimization, 24(4):760-770, 1986.

[12] Y. Hong. $\mathrm{H}_{\infty}$ control, stabilization, and input-output stability of nonlinear systems with homogeneous properties. Automatica, 37(7):819829, 2001.

[13] Y. Hong, J. Huang, and Y. Xu. On an output feedback finitetime stabilization problem. IEEE Transactions on Automatic Control, 46(2):305 - 309, 2001

[14] A. Levant. Homogeneity approach to high-order sliding mode design Automatica, 41:823-830, 2005.

[15] S. Li, H. Sun, J. Yang, and X. Yu. Continuous finite-time output regulation for disturbed systems under mismatching condition. IEEE Transactions on Automatic Control, 60(1):277-282, Jan 2015.

[16] J. A. Moreno. A Lyapunov approach to output feedback control using second-order sliding modes. IMA Journal of Mathematical Control and Information, 29(3):291 -308, 2012.

[17] J. A. Moreno and M. Osorio. Strict Lyapunov functions for the super-twisting algorithm. IEEE Transactions on Automatic Control, 57:1035-1040, 2012.

[18] Y. Orlov. Finite Time Stability and Robust Control Synthesis of Uncertain Switched Systems. SIAM Journal of Control and Optimization, 43(4):1253-1271, 2005.

[19] Y. Orlov, Y. Aoustin, and C. Chevallereau. Finite time stabilization of a perturbed double integrator part I: Continuous sliding mode-based output feedback synthesis. IEEE Transactions on Automatic Control, 56(3):614-618, March 2011.

[20] H. B. Oza, Y. V. Orlov, and S. K. Spurgeon. On robustness of a class of homogeneous continuous finite time controllers. In IEEE Conference on Decision and Control (CDC), pages 6279-6284, Dec 2014.

[21] H.B. Oza, Y.V. Orlov, and S.K. Spurgeon. Continuous uniform finite time stabilization of planar controllable systems. Accepted for publiaciton in SIAM Journal on Control and Optimization, 2015.

[22] W. Perruquetti and T. Floquet. Homogeneous finite time observer for nonlinear systems with linearizable error dynamics. In IEEE Conference on Decision and Control, New Orleans, Louisiana, U.S.A., 2007.

[23] A. Polyakov, D. Efimov, and W. Perruquetti. Finite-time and fixedtime stabilization: Implicit Lyapunov function approach. Automatica, 51:332-340, 2015.

[24] A. Polyakov and L. Fridman. Stability notions and Lyapunov functions for sliding mode control systems. Journal of the Franklin Institute, 351(4):1831-1865, 2014.

[25] A. Polyakov and J.-P. Richard. Suboptimal LMI-based solution of minimum time control problem. In Proceedings of 19th IFAC World Congress, pages 2475-2480, 2014.

[26] E. Roxin. On finite stability in control systems. Rendiconti del Circolo Matematico di Palermo, 15:273-283, 1966.

[27] V.I. Zubov. On systems of ordinary differential equations with generalized homogenous right-hand sides. Izvestia vuzov. Mathematica. (in Russian), 1:80-88, 1958. 